\title{
Review on CPT and decoherence measurements with Kaons
}

\author{
Marianna Testa* \\ Rome Univ. "La Sapienza" and INFN \\ E-mail: marianna.testa@roma1.infn.it
}

Neutral kaons provide one of the most sensitive systems to test quantum mechanics and $C P T$ violation. In the first part of this report I review the results on $C P T$ violation searches in the kaon system from the CPLEAR, KLOE and KTeV experiments. In the second part, I report tests of quantum coherence related to $C P T$ violation, performed at CPLEAR and KLOE by studying the time evolution of the kaon system. The results show no deviations from the expectations of quantum mechanics and CPT symmetry, and the accuracy reaches the interesting region of the Planck's scale.

\section{KAON International Conference}

May 21-25 2007

Laboratori Nazionali di Frascati dell'INFN, Rome, Italy

\footnotetext{
* Speaker.
} 


\section{Introduction}

The time evolution of the neutral kaon system is described by

$$
i \frac{\partial}{\partial t}\left(\begin{array}{c}
K^{0} \\
\bar{K}^{0}
\end{array}\right)=\left(M-\frac{i}{2} \Gamma\right)\left(\begin{array}{c}
K^{0} \\
\bar{K}^{0}
\end{array}\right),
$$

where $M$ and $\Gamma$ are the $2 \times 2$ mass and decay matrices.

$C P T$ violation in the time evolution is parametrized by

$$
\delta=\frac{i\left(M_{K^{0}}-M_{\bar{K}^{0}}\right)+1 / 2\left(\Gamma_{K^{0}}-\Gamma_{\bar{K}^{0}}\right)}{\Gamma_{S}-\Gamma_{L}} \cos \left(\phi_{S W}\right) e^{i \phi_{S W}}
$$

where $\phi_{S W}$ is the superweak phase, defined by $\tan \phi_{S W}=2\left(M_{S}-M_{L}\right) /\left(\Gamma_{S}-\Gamma_{L}\right)$ and $M_{S, L}$ and $\Gamma_{S, L}$ are the mass and decay width of $K_{S, L}$.

The $\delta$ parameter has been directly determined by measuring the time-dependent semileptonic asymmetry, and has been indirectly measured by comparing the $K_{S}$ and $K_{L}$ semileptonic asymmetry and using the Bell-Steinberger relation (sec. 2, 3).

$C P T$ violation has been also searched for in the kaon decay to $\pi^{+} \pi^{-}$and in semileptonic decay. $C P T$ tests in the decay to $\pi^{+} \pi^{-}$are based on the phase differences $\phi_{+-}-\phi_{S W}$ and $\phi_{00}-\phi_{+-}$, where $\left|\eta_{+-(00)}\right| e^{i \phi_{+-(00)}}=\frac{A\left(K_{L} \rightarrow \pi^{+(0)} \pi^{-(0)}\right)}{A\left(K_{S} \rightarrow \pi^{+(0)} \pi^{-(0)}\right)}$ and have been performed measuring interference patterns (sec. 円).

Semileptonic amplitudes are parametrized by $x_{+}$, describing violation of the $\Delta S=\Delta Q$ rule, and $x_{-}$ and $y$, describing $C P T$ violation in the $\Delta S \neq \Delta Q$ and $\Delta S=\Delta Q$ decay amplitudes, respectively. The $x_{+,-}$and $y$ parameters have been obtained by measuring the semileptonic asymmetries (sec. 2).

Different experimental approaches giving access to complementary information have been used to test $C P T$ symmetry in the kaon system: tagging of kaon strangeness at CPLEAR, regenerator technique at $\mathrm{KTeV}$ and coherent $K_{S} K_{L}$ production at KLOE.

\section{CPT tests at CPLEAR}

The CPLEAR experiment used low-energy antiprotons stopped in a gaseous hydrogen target. Single and two neutral kaons were provided by the processes:

$$
\begin{aligned}
p \bar{p} \rightarrow & K^{0} K^{-} \pi^{+}, \bar{K}^{0} K^{+} \pi^{-} \\
& K^{0} \bar{K}^{0} .
\end{aligned}
$$

In latter reaction the $K^{0} \bar{K}^{0}$ system is mostly produced in a $J^{P C}=1^{--}$state [1]:

$$
|i\rangle \propto\left|K^{0}(+\vec{p})\right\rangle\left|\bar{K}^{0}(-\vec{p})\right\rangle-\left|\bar{K}^{0}(+\vec{p})\right\rangle\left|K^{0}(-\vec{p})\right\rangle
$$

A direct measurement of $\Re \delta$ has been performed at CPLEAR using the reaction 2.1, by measuring the time-dependent semileptonic asymmetry [1]:

$$
A_{\delta}(\tau)=\frac{\bar{R}_{+}(\tau)-R_{-}(\tau)}{\bar{R}_{+}(\tau)+R_{-}(\tau)}+\frac{\bar{R}_{-}(\tau)-R_{+}(\tau)}{\bar{R}_{-}(\tau)+R_{+}(\tau)}=4 \mathfrak{R} \delta+\mathscr{F}\left(\mathfrak{I} \delta, y, \mathfrak{R} x_{-}, \mathfrak{I} x_{+}\right)
$$


where $R_{\mp}(\tau)\left(\bar{R}_{\mp}(\tau)\right)$ is the decay rate at proper time $\tau$ to $e^{\mp} \pi^{ \pm} v$ for events tagged as $K^{0}\left(\bar{K}^{0}\right)$ at $\tau=0$. The asymmetry depends, beside the $C P T$-violating parameter $\delta$, on the parameters $y$ and $x_{ \pm}$. The strangeness of the kaon at $\tau=0$ is identified by the accompanying charged kaon. The measured asymmetry is shown in fig. 1 1 and the results are:

$$
\begin{aligned}
\mathfrak{R} \delta & =(0.30 \pm 0.33 \pm 0.06) \times 10^{-3} \\
\mathfrak{I} \delta & =(-1.5 \pm 2.3 \pm 0.3) \times 10^{-2} \\
\mathfrak{R} x_{-} & =(0.2 \pm 1.3 \pm 0.3) \times 10^{-2} \\
\mathfrak{I} x_{+} & =(1.2 \pm 2.2 \pm 0.3) \times 10^{-2}
\end{aligned}
$$

consistent with $C P T$ symmetry.

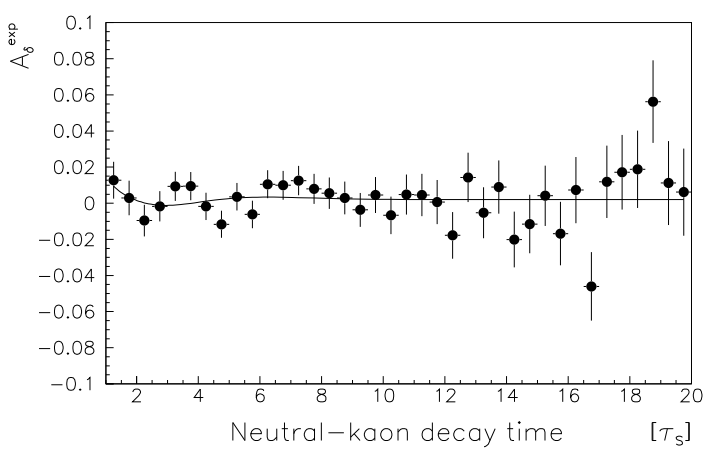

Figure 1: Semileptonic asymmetry as a function of the kaon decay times (in $\tau_{S}$ units). The solid line represents the fit result.

This latter result is improved by adding as a constraint the KLOE measurement [2] $A_{S}-A_{L}=$ $4\left[\Re \delta+\Re x_{-}\right]=(-1.8 \pm 10.0) \times 10^{-3}$ to the original CPLEAR fit, obtaining $\mathfrak{I} x_{+}=(0.8 \pm 0.7) \times$ $10^{-2}$, whose reduced uncertainty improves the determination of $\mathfrak{I} \delta$ and $\mathfrak{R} \varepsilon$ through the BellSteinberger relation (3.2) (sec. 3.1)

\section{CPT tests at KLOE}

The KLOE detector operates at the Frascati $\phi$-factory DA $\Phi \mathbf{N E}$, an $e^{+} e^{-}$collider working at the center of mass energy $W \sim m_{\phi} \sim 1.02 \mathrm{GeV}$. The $K$ mesons are produced from the $\phi$ decay in a $J^{P C}=1^{--}$state:

$$
\begin{aligned}
|i\rangle & \propto\left|K^{0}(+\vec{p})\right\rangle\left|\bar{K}^{0}(-\vec{p})\right\rangle-\left|\bar{K}^{0}(+\vec{p})\right\rangle\left|K^{0}(-\vec{p})\right\rangle \\
& \propto\left|K_{S}(+\vec{p})\right\rangle\left|K_{L}(-\vec{p})\right\rangle-\left|K_{L}(+\vec{p})\right\rangle\left|K_{S}(-\vec{p})\right\rangle
\end{aligned}
$$

so that observation of a $K_{S}$ in an event signals the presence of a $K_{L}$ and vice-versa. In particular, the unique possibility to select a pure and monochromatic $K_{S}$ beam, not possible in a fixed target experiment, allows to perform $C P T$ tests by measuring the semileptonic charge asymmetry: $A_{S, L}=\frac{\Gamma\left(K_{S, L} \rightarrow \pi^{-} e^{+} v\right)-\Gamma\left(K_{S, L} \rightarrow \pi^{+} e^{-} \bar{v}\right)}{\Gamma\left(K_{S, L} \rightarrow \pi^{-} e^{+} v\right)+\Gamma\left(K_{S, L} \rightarrow \pi^{+} e^{-} \bar{v}\right)}$. The difference between the charge asymmetries 
$A_{S}-A_{L}=4\left(\Re \delta+\Re x_{-}\right)$signals $C P T$ violation either in the mass matrix or in the decay amplitudes with $\Delta S \neq \Delta Q$, while the sum $A_{S}+A_{L}=4(\Re \varepsilon-\Re y)$ allows to test $C P T$ violation in the $\Delta S=\Delta Q$ decay amplitude. $A_{S}$ has been measured for the first time by KLOE [2]. Its value, $A_{S}=(1.5 \pm 9.6 \pm 2.9) \times 10^{-3}$, is compatible with the measurement of $A_{L}$ [B]. By comparing $A_{S}$ and $A_{L}$, KLOE has also obtained $\Re x_{-}=(-0.8 \pm 2.4 \pm 0.7) \times 10^{-3}$ and $\Re y=(0.4 \pm 2.4 \pm 0.7) \times 10^{-3}$. The results are consistent with $C P T$ symmetry.

\subsection{Bell Steinberger Relation}

The most powerful test of $C P T$ invariance in the neutral kaon system is presently obtained by means of the Bell-Steinberger relation [ [ and $\Re \varepsilon$, to the decay amplitudes of $K_{L}$ and $K_{S}$ into the same final state:

$$
\left[\frac{\Gamma_{S}+\Gamma_{L}}{\Gamma_{S}-\Gamma_{L}}+i \tan \phi_{S W}\right] \frac{\Re \varepsilon-i \mathfrak{l} \delta}{1+|\varepsilon|^{2}}=\frac{1}{\Gamma_{S}-\Gamma_{L}} \sum_{f} a_{S}^{*}(f) a_{L}(f)=\sum_{f} \alpha_{f}
$$

where $a_{S, L}(f)$ are the $K_{S, L}$ decay amplitudes to the final state $f$. KLOE has recently determined $\Re \varepsilon$ and $\mathfrak{I} \delta$ from (3.2). Details of the experimental inputs can be found in ref. [5]. The main improvements are due to the KLOE upper limit on the $\operatorname{BR}\left(K_{S} \rightarrow \pi^{0} \pi^{0} \pi^{0}\right)$ [6] and the KLOE measurement of the semileptonic $K_{S}$ charge asymmetry $A_{S}$ [饭, which allows to calculate the semileptonic contribution $\alpha_{k l 3}=2 \tau_{K_{S}} / \tau_{K_{L}} B(k l 3)\left(\Re \varepsilon-\Re y-i\left(\mathfrak{I} \delta+\mathfrak{I} x_{+}\right)\right)=2 \tau_{K_{S}} / \tau_{K_{L}} B(k l 3)\left(\left(A_{S}+A_{L}\right) / 4-i(\mathfrak{I} \delta+\right.$ $\left.\left.\mathfrak{I} x_{+}\right)\right)$independently from the $y$ parameter and to determine $\mathfrak{I} x_{+}$from a combined fit of $A_{S}$ with the semileptonic time dependent decay rate asymmetry measured by CPLEAR [1] (sec. 2).

The results are $\operatorname{Re}(\varepsilon)=(159.6 \pm 1.3) \times 10^{-5}$ and $\operatorname{Im}(\delta)=(0.4 \pm 2.1) \times 10^{-5}$, resulting in an improvement to the CPLEAR measurement [1]: $\operatorname{Re}(\varepsilon)=(164.9 \pm 2.5) \times 10^{-5}$ and $\operatorname{Im}(\delta)=(2.4 \pm$ $5.0) \times 10^{-5}$.

The limits on $\mathfrak{I} \delta$ and $\Re \delta$ can be used to constrain the mass and width difference between $K^{0}$ and
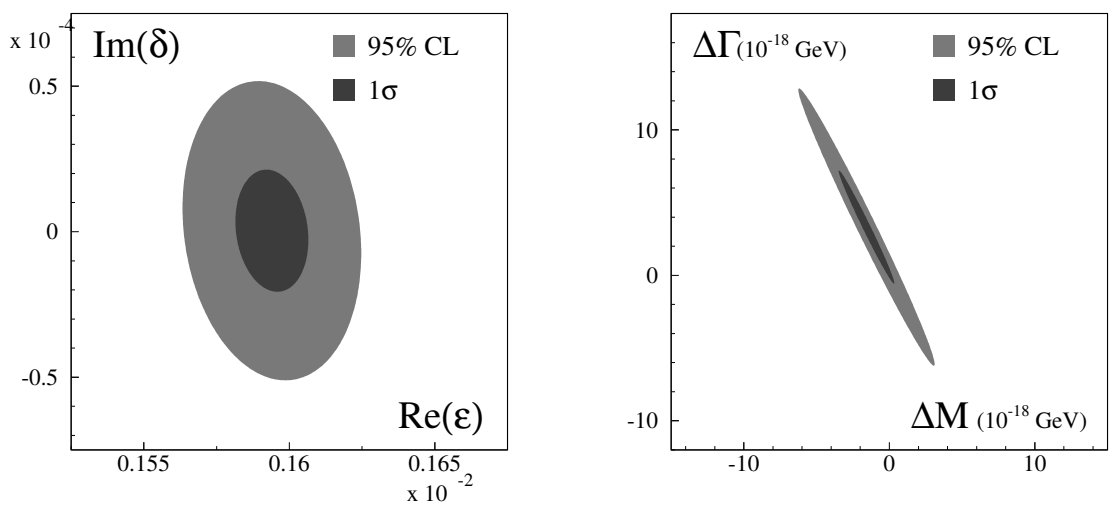

Figure 2: Allowed region at $68 \%$ and $95 \% \mathrm{CL}$ in the $\Re \varepsilon, \Im \delta$ plane and in the $\Delta M, \Delta \Gamma$ plane.

$\bar{K}^{0}$ from eq.1.2. The allowed region in the $\left(M_{K^{0}}-M_{\bar{K}^{0}}\right),\left(\Gamma_{K^{0}}-\Gamma_{\bar{K}^{0}}\right)$ plane is shown in the right panel of fig. 3.1. Assuming no $C P T$ violation in kaon decay (i. e. $\Gamma_{K^{0}}=\Gamma_{\bar{K}^{0}}$ ) KLOE has obtained the following best limits on the neutral kaon mass difference: 


$$
-5.3 \times 10^{-19}<M_{K^{0}}-M_{\bar{K}^{0}}<6.3 \times 10^{-19} \mathrm{GeV} \quad \text { at } 95 \% \text { C.L. }
$$

\section{CPT tests at KTeV}

The $\mathrm{KTeV}$ experiment used a regenerator to provide a source of $K_{S}$ decays . The $K_{S}-K_{L}$ interference pattern downstream the regenerator allows to perform $C P T$ tests based on phase measurements. The $\phi_{+-}, \phi_{00}$ and $\phi_{\mathrm{SW}}$ phases have been measured by fitting the time decay distribution of $K_{S, L} \rightarrow \pi^{+} \pi^{-}$and $\pi^{0} \pi^{0}$ events in the regenerator beam decay (fig. 3 ) with

$$
R(t) \propto\left(|\eta|^{2} e^{-t / \tau_{L}}+|\rho|^{2} e^{-t / \tau_{S}}+2|\rho||\eta| \cos \left(\Delta m t+\phi_{\rho}-\phi_{\eta}\right) e^{-t /\left(\tau_{S}+\tau_{L}\right)}\right)
$$

where $\rho=|\rho| e^{i \phi_{\rho}}$ is the coherent regeneration amplitude and $\eta=\eta_{+-, 00}$.

The results are[7]:

$$
\begin{aligned}
\phi_{+-}-\phi_{S W} & =+0.61 \pm 0.62 \pm 1.01 \\
\phi_{00}-\phi_{+-} & =+0.39 \pm 0.22 \pm 0.45
\end{aligned}
$$

where the latter result benefits from the cancellation of uncertainties due to the regeneration phase. Both results are consistent with $C P T$ symmetry.

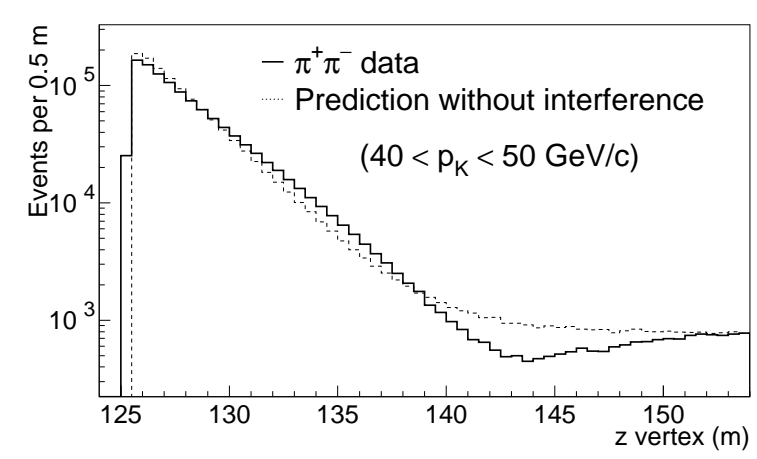

Figure 3: $z$ decay distribution of $K \rightarrow \pi^{+} \pi^{-}$in the regenerator beam for data (black point) and MC (dashed) without the interference term.

\section{Tests of quantum coherence}

Tests of quantum coherence have been performed at CPLEAR and KLOE by studying the time evolution of the entangled states (2.3) and (3.1), respectively. In particular, according to quantum mechanics $(\mathrm{QM})$ the double differential time distribution into final states $f_{1}$ and $f_{2}$ at proper times $t_{1}$ and $t_{2}$ for the state (3.1) is

$$
\begin{aligned}
I\left(f_{1}, t_{1} ; f_{2}, t_{2}\right) \propto & \left|\eta_{1}\right|^{2} e^{-\Gamma_{L} t_{1}-\Gamma_{S} t_{2}}+\left|\eta_{2}\right|^{2} e^{-\Gamma_{S} t_{1}-\Gamma_{L} t_{2}} \\
& -2\left|\eta_{1}\right|\left|\eta_{2}\right| e^{-\left(\Gamma_{S}+\Gamma_{L}\right)\left(t_{1}+t_{2}\right) / 2} \cos \left[\Delta m\left(t_{1}-t_{2}\right)+\phi_{2}-\phi_{1}\right]
\end{aligned}
$$


One of the most direct ways to search for deviations from QM is to introduce a decoherence parameter $\zeta[8]$, i.e. multiplying by a factor $(1-\zeta)$ the interference term in (5.1). The definition of $\zeta$ depends on the basis in which the initial state (3.1) is written [⿰月]. The case $\zeta=1$ (i.e. total decoherence) corresponds to the spontaneous factorization of states (known as Furry's hypothesis [10]).

At CPLEAR the spontaneous factorization of the state (2.3) has been tested by measuring the asymmetry between the intensities for like- and unlike strangeness kaons as a function of the kaon decay times []]. According to QM, this asymmetry is given by

$$
A\left(t_{1}, t_{2}\right)=\frac{2 e^{\left(\Gamma_{S}+\Gamma_{L}\right)\left(t_{1}+t_{2}\right) / 2} \cos \left(\Delta m\left(t_{1}-t_{2}\right)\right)}{e^{-\Gamma_{S} t_{1}-\Gamma_{L} t_{2}}+e^{-\Gamma_{S} t_{2}-\Gamma_{L} t_{1}}}
$$

while according to the separability hypothesis $A\left(t_{1}, t_{2}\right)=0$. The kaon strangeness is determined via strong interaction processes $K^{0}+N \rightarrow K^{+}+X, \bar{K}^{0}+N \rightarrow K^{-}+X$ and $\bar{K}^{0}+N \rightarrow \Lambda\left(\rightarrow p+\pi^{-}\right)+X$ with two absorbers, placed around the target. The like (unlike) strangeness events are defined as $K^{-} \Lambda\left(K^{+} \Lambda\right)$ events. The asymmetry is measured in two configurations: in the first (second) one the time difference of the interaction is $t_{1}-t_{2} \simeq 0\left(t_{1}-t_{2} \simeq 1.2 \tau_{S}\right)$. The results are shown in fig. 7 . The measured asymmetries are consistent with QM predictions and the separability hypothesis is

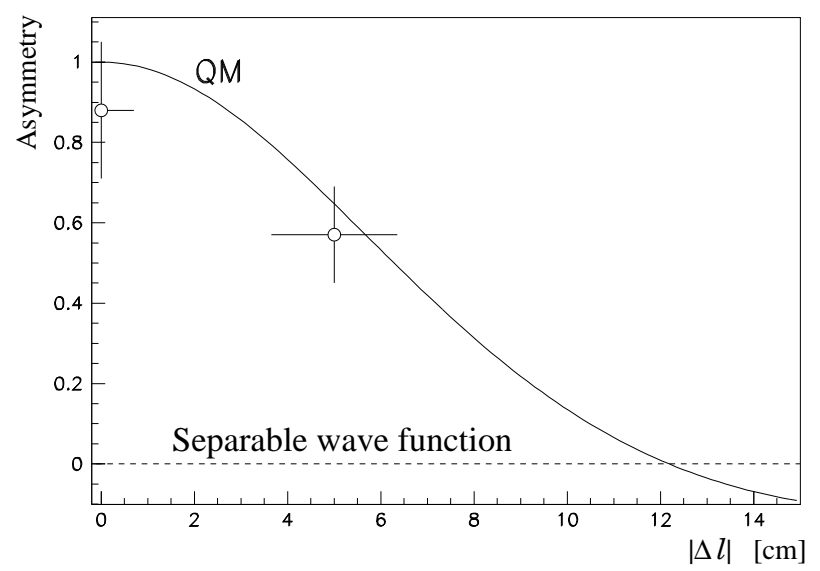

Figure 4: Asymmetry for like- and unlike strangeness events in the two experimental configurations. The solid and dashed curve represent the QM prediction and that for a separable wave function, respectively.

excluded with $C L>99.99 \%$.

CPLEAR data have been re-analysed by Bertlmann et al. [9, 11] to measure the decoherence parameter $\zeta$. From the fit to the asymmetry data, they found $\zeta_{S, L}=0.13_{-0.15}^{+0.16}$ and $\zeta_{0,0}=0.4 \pm 0.7$ in the $K_{S} K_{L}$ and $K^{0} \bar{K}^{0}$ basis respectively.

In the KLOE experiment, tests of QM have been performed by studying the interference pattern of the decay $K_{L} K_{S} \rightarrow \pi^{+} \pi^{-} \pi^{+} \pi^{-}[12]$. The decoherence parameter $\zeta$ has been measured by fitting the distribution $I\left(\Delta t ; \pi^{+} \pi^{-}, \pi^{+} \pi^{-}\right)$, where $\Delta t$ is the difference between the proper time of the $K_{S}$ and $K_{L}$ decays into the final state $\pi^{+} \pi^{-}, \pi^{+} \pi^{-}$. The fit of the $\Delta t$ distribution is shown in fig. 5: the peak at $\Delta t \sim 17 \tau_{S}$ is due to the coherent and incoherent regeneration on the spherical beam pipe. The results are $\zeta_{S, L}=0.018 \pm 0.040 \pm 0.007$ and $\zeta_{0,0}=(0.10 \pm 0.21 \pm 0.04) \times 10^{-5}$. 


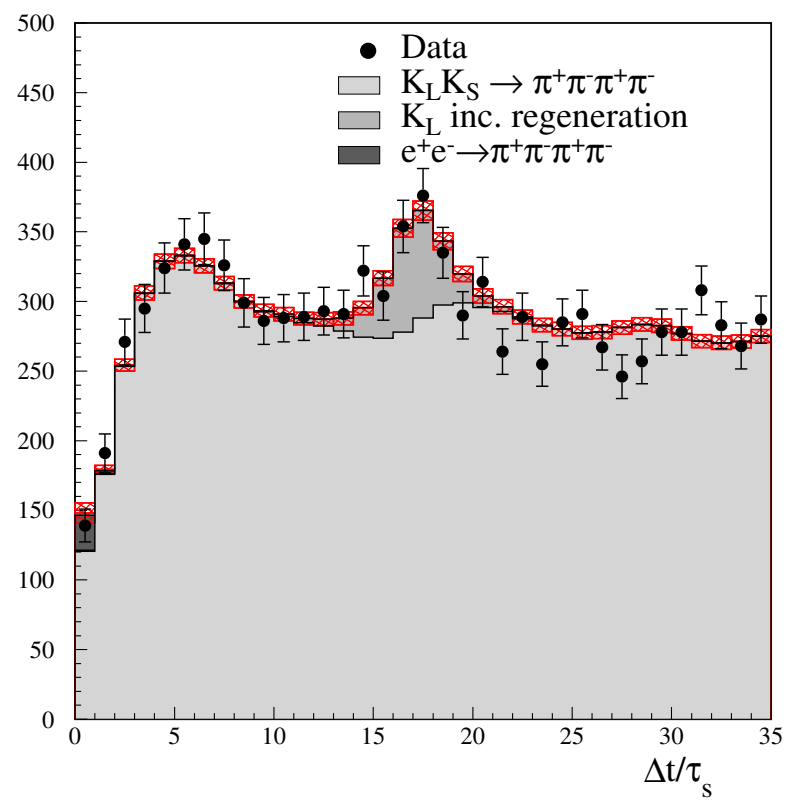

Figure 5: Fit of the $\Delta t$ distribution. The black points with errors are data and the solid histogram is the fit result to obtain $\zeta_{S, L}$. The uncertainty arising from the efficiency correction is shown as the hatched area. The peak at $\Delta t \sim 17 \tau_{S}$ is due to the coherent and incoherent regeneration on the beam pipe.

The results are consistent with QM. Since decoherence in the $K^{0} \bar{K}^{0}$ basis would result in the $C P$ allowed $K_{S} K_{S} \rightarrow \pi^{+} \pi^{-} \pi^{+} \pi^{-}$decays, the value for $\zeta_{0,0}$ is naturally much smaller.

\section{Decoherence and CPT violation due to quantum gravity effects}

In a quantum gravity framework, space-time fluctuations at the Planck scale $\left(\sim 10^{-33} \mathrm{~cm}\right)$, might induce a pure state to evolve into a mixed one [13]. This decoherence, in turn, necessarily implies $C P T$ violation [14]. $\mathrm{QM}$ and $C P T$ violation related to this decoherence mechanism could be observed in the decay time distribution of the $K^{0} \bar{K}^{0}$ system [15]. In ref. [15] three new $C P T$ and QM-violating real parameters $\alpha, \beta$ and $\gamma$ having mass dimension are introduced to parametrize the decoherence effects. They satisfy the conditions $\alpha, \gamma>0$ and $\alpha \gamma>\beta^{2}$ and are expected to be at most $\mathscr{O}\left(M_{K}^{2} / M_{P}\right) \sim 2 \times 10^{-20} \mathrm{GeV}\left[15\right.$, 16], where $M_{P}=1 / \sqrt{G_{N}}=1.22 \times 10^{19} \mathrm{GeV}$ is the Planck scale. For an entangled system the further conditions $\alpha=\gamma$ and $\beta=0$ (complete positivity of the density matrix) ensure the conservation of probability [17, 18, 19].

At CPLEAR measurements of the $\alpha, \beta$ and $\gamma$ parameters have been obtained using the time evolution of single kaons from the processes (2.1] [20]. The strangeness of the kaon is identified by the accompanying charged kaon. A combined fit of the asymmetries obtained from the time distributions of the $K^{0}\left(\bar{K}^{0}\right) \rightarrow \pi^{+} \pi^{-}$and $K^{0}\left(\bar{K}^{0}\right) \rightarrow e^{\mp} \pi^{ \pm} v$ decays gives $\alpha=(-0.5 \pm 2.8) \times$ $10^{-17} \mathrm{GeV}, \beta=(2.5 \pm 2.3) \times 10^{-19} \mathrm{GeV}$ and $\gamma=(1.1 \pm 2.5) \times 10^{-21} \mathrm{GeV}$. The fit to the asymmetry obtained with $K^{0}\left(\bar{K}^{0}\right) \rightarrow \pi^{+} \pi^{-}$decays is shown in fig. 6.

At KLOE the $\gamma$ parameter has been measured for the first time in the entangled kaon state by fitting the $I\left(\Delta t ; \pi^{+} \pi^{-} \pi^{+} \pi^{-}\right)$distribution [12]. The result is: $\gamma=\left(1.1_{-2.4}^{+2.9} \pm 0.4\right) \times 10^{-21} \mathrm{GeV}$, 


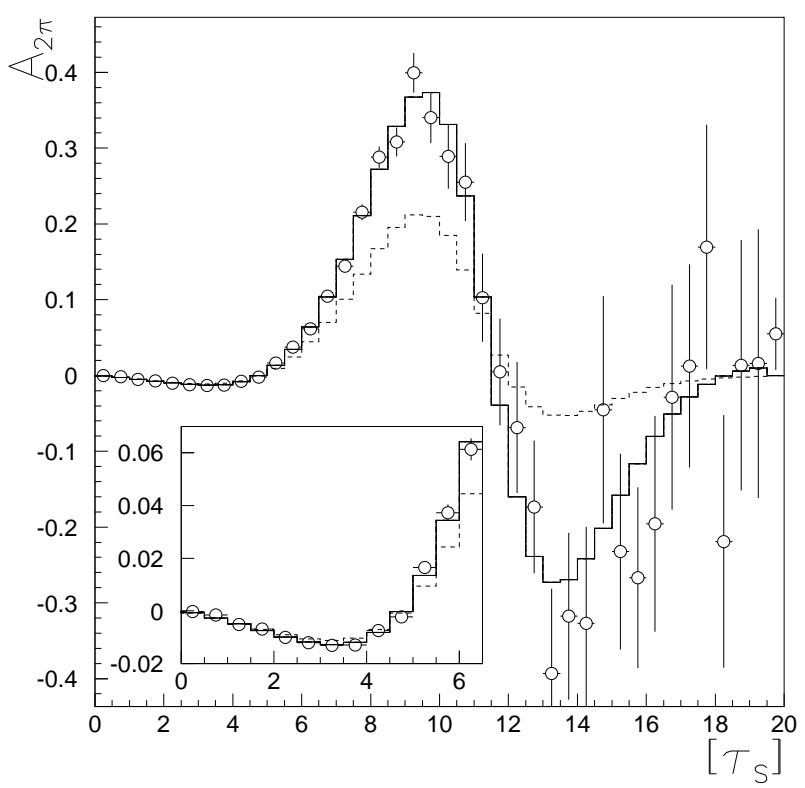

Figure 6: Asymmetry for $K^{0}\left(\bar{K}^{0}\right) \rightarrow \pi^{+} \pi^{-}$events. The solid (dashed) line represents the fit result (expectation with $\alpha=4 \times 10^{-16} \mathrm{GeV}, \beta=2.3 \times 10^{-18} \mathrm{GeV}$ and $\gamma=3.7 \times 10^{-20} \mathrm{GeV}$ )

competitive with that obtained by CPLEAR using single kaon beams.

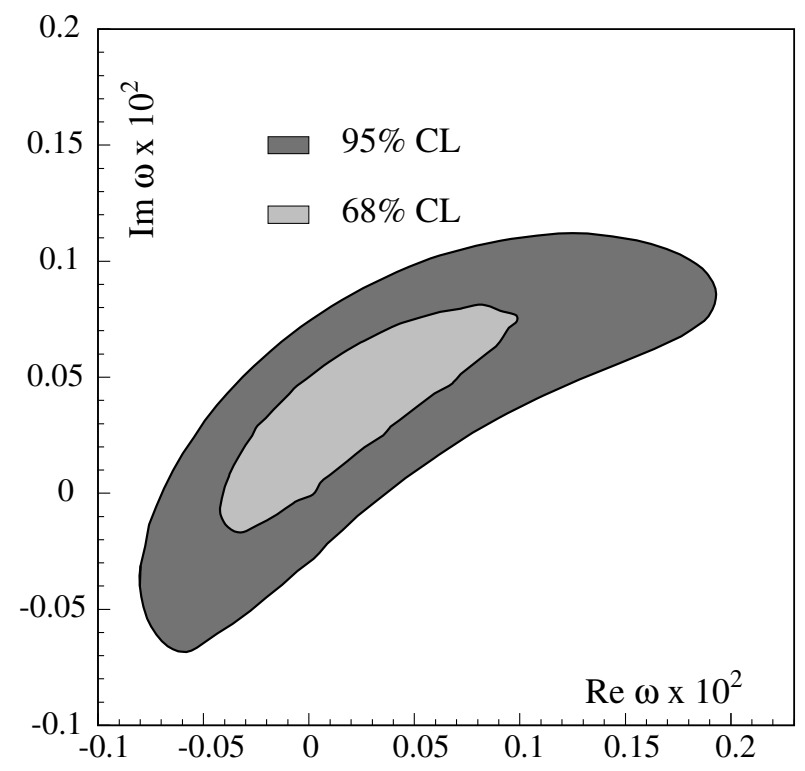

Figure 7: Contour plot of $\mathfrak{I} \omega$ versus $\Re \omega$ at $68 \%$ and $95 \%$ C.L.

A new type of $C P T$ violation for correlated kaon states is also expected in this context [21, 22]. As a result, the initial entangled state (3.1) can be parametrized in general as:

$$
|i\rangle \propto\left|K^{0}(+\vec{p})\right\rangle\left|\bar{K}^{0}(-\vec{p})\right\rangle-\left|\bar{K}^{0}(+\vec{p})\right\rangle\left|K^{0}(-\vec{p})\right\rangle
$$




$$
\begin{aligned}
& +\omega\left(\left|K^{0}(+\vec{p})\right\rangle\left|\bar{K}^{0}(-\vec{p})\right\rangle+\left|\bar{K}^{0}(+\vec{p})\right\rangle\left|K^{0}(-\vec{p})\right\rangle\right) \\
\propto & \left|K_{S}(+\vec{p})\right\rangle\left|K_{L}(-\vec{p})\right\rangle-\left|K_{L}(+\vec{p})\right\rangle\left|K_{S}(-\vec{p})\right\rangle \\
& +\omega\left(\left|K_{S}(+\vec{p})\right\rangle\left|K_{S}(-\vec{p})\right\rangle-\left|K_{L}(+\vec{p})\right\rangle\left|K_{L}(-\vec{p})\right\rangle\right)
\end{aligned}
$$

where $\omega$ is a complex parameter describing $C P T$ violation. Its order of magnitude might be at most $|\omega| \sim \sqrt{\left(M_{K}^{2} / M_{P}\right) / \Delta \Gamma} \sim 10^{-3}$, with $\Delta \Gamma=\Gamma_{S}-\Gamma_{L}$.

At KLOE the complex parameter $\omega$ has been measured for the first time [12]. The $K_{S} K_{L} \rightarrow$ $\pi^{+} \pi^{-} \pi^{+} \pi^{-}$decay is the most sensitive channel to search for this kind of $C P T$ violation, as the leading $K_{S} K_{L}$ terms are $C P$ suppressed (eq. 6.1)

The results, obtained by fitting the $I\left(\Delta t ; \pi^{+} \pi^{-} \pi^{+} \pi^{-}\right)$distribution, are $\Re \omega=\left(1.1_{-5.3}^{+8.7} \pm 0.9\right) \times$ $10^{-4}$ and $\Im \omega=\left(3.4_{-5.0}^{+4.8} \pm 0.6\right) \times 10^{-4}$, consistent with QM and $C P T$ symmetry. The regions allowed at 68 and 95\% C.L. in the plane $(\mathfrak{I} \omega, \Re \omega)$ are shown in fig. 7. The upper limit $|\omega|<$ $2.1 \times 10^{-3}$ at $95 \%$ C.L. is obtained.

\section{References}

[1] CPLEAR Collaboration, Phys. Reports 374, 165 (2003)

[2] KLOE Collaboration, Phys. Lett. B 636, 173 (2006)

[3] Particle Data Group, W.-M. Yao et al., Journal of Physics G33, 1 (2006)

[4] J.S. Bell and J. Steinberger, Proc. of Oxford Int. Conf. on Elementary Part., 195 (1965)

[5] KLOE Collaboration, JHEP 0612, 011 (2006)

[6] KLOE collaboration, Phys. Lett. B 619, 61 (2005)

[7] KTeV Collaboration, Phys. Rev. D 67, 012005 (2003)

[8] P. H. Eberhard, "Tests of Quantum Mechanics at a $\phi$ factory" in The second DaФne handbook, ed. L. Maiani, G. Pancheri, N. Paver, Vol.I

[9] R. A. Bertlmann et al., Phys. Rev. D 60, 114032 (1999)

[10] W. H. Furry, Phys. Rev. D 49, 393 (1936)

[11] R. A. Bertlmann et al., Phys. Rev. A 68, 012111 (2003)

[12] KLOE Collaboration, Phys. Lett. B 642, 315 (2006)

[13] S. Hawking, Commun. Math. Phys. 87, 395 (1982)

[14] R. Wald, Phys. Rev. D 21, 2742 (1980)

[15] J. Ellis, J. S. Hagelin, D. V. Nanopoulos, M. Srednicki, Nucl. Phys. B 241, 381 (1984)

[16] J. Ellis, J. L. Lopez, N. .E. Mavromatos, D. V. Nanopoulos, Phys. Rev. D 53, 3846 (1996)

[17] P. Huet, M. Peskin, Nucl. Phys. B 434, 3 (1995)

[18] F. Benatti, R. Floreanini, Nucl. Phys. B 511, 550 (1998)

[19] F. Benatti, R. Floreanini, Phys. Lett. B 468, 287 (1999)

[20] CPLEAR Collaboration, Phys. Lett. B 364, 239 (1995)

[21] J. Bernabeu, N. Mavromatos, J. Papavassiliou, Phys. Rev. Lett. 92, 131601 (2004)

[22] J. Bernabeu, N. Mavromatos, J. Papavassiliou, A. Waldron-Lauda, Nucl. Phys. B 744, 180 (2006) 